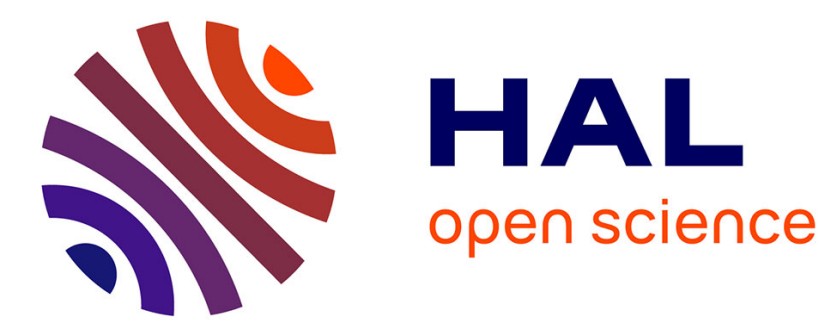

\title{
On input-to-output stability and robust synchronization of generalized Persidskii systems
}

Wenjie Mei, Denis Efimov, Rosane Ushirobira

\section{To cite this version:}

Wenjie Mei, Denis Efimov, Rosane Ushirobira. On input-to-output stability and robust synchronization of generalized Persidskii systems. IEEE Transactions on Automatic Control, In press. hal03384183

\section{HAL Id: hal-03384183 \\ https://hal.inria.fr/hal-03384183}

Submitted on 18 Oct 2021

HAL is a multi-disciplinary open access archive for the deposit and dissemination of scientific research documents, whether they are published or not. The documents may come from teaching and research institutions in France or abroad, or from public or private research centers.
L'archive ouverte pluridisciplinaire HAL, est destinée au dépôt et à la diffusion de documents scientifiques de niveau recherche, publiés ou non, émanant des établissements d'enseignement et de recherche français ou étrangers, des laboratoires publics ou privés. 


\title{
On input-to-output stability and robust synchronization of generalized Persidskii systems
}

\author{
Wenjie Mei*, Denis Efimov* ${ }^{* \dagger}$, Rosane Ushirobira*
}

\begin{abstract}
In this paper, we study a class of generalized Persidskii systems with external disturbances and establish conditions, in the form of linear matrix inequalities, for input-to-output stability (IOS) and robust synchronization for these systems. We apply the obtained results to the robust control design for synchronizing linear systems and to the synchronization of Hindmarsh-Rose models of neurons.
\end{abstract}

Index Terms-Input-to-output stability, robust synchronization, generalized Persidskii systems.

\section{INTRODUCTION}

Stability analysis for dynamical systems is a complicated problem, especially in the nonlinear case and in the presence of external perturbations [2]-[4]. One of the most general frameworks in this domain is given by the input-to-output stability (IOS) concept [5], [6], which quantifies the boundedness and the convergence of an output signal for a nonlinear dynamical system in the presence of essentially bounded exogenous inputs. This theory also provides necessary and sufficient conditions for IOS in terms of the existence of corresponding Lyapunov functions [6]. The well-known inputto-state stability (ISS) property [7] is a particular case of IOS when the whole state is considered as the system output. The main drawback of applying these concepts is the lack of constructive methods to design the related Lyapunov functions.

Most existing approaches for synthesizing Lyapunov functions for nonlinear dynamics involve various canonical forms such as Lur'e systems [8], Lipschitz dynamics, Persidskii systems [9], and homogeneous models. In the present paper, we focus our attention on Persidskii systems, which have been extensively studied in the context of neural networks [10] and power systems [11]. This class of models was introduced in [9], [12], and recently the ISS conditions have been established in [13] for its generalized form. The properties of the Lyapunov function proposed in [13] and its time derivative are analyzed using linear matrix inequalities (LMI), which is a rare case for nonlinear systems taking into account a possible highly nonlinear nature of generalized Persidskii systems. The main goal of our work is to extend those results to input-tooutput stability (IOS) and apply them to study synchronization conditions for a family of generalized Persidskii systems.

\footnotetext{
* Inria, Univ. Lille, CNRS, UMR 9189 - CRIStAL, F-59000 Lille, France. $\dagger$ ITMO University, 49 av. Kronverkskiy, 197101 Saint Petersburg, Russia. This work was partially supported by the China Scholarship Council (CSC) Scholarship (CSC No. 201908050104), and by the Ministry of Science and Higher Education of Russian Federation, passport of goszadanie no. 20190898.

A preliminary conference version of this work [1] mainly focuses on the synchronization application, and the IOS conditions are only briefly discussed (solely a disturbance-free case is studied).
}

Synchronization is a complex phenomenon that is frequently observed in networked and interconnected systems. It has been extensively investigated in various fields, e.g., robotics, communication security, autonomous driving [14]-[17]. The principal methods to achieve synchronization for nonlinear systems are based on the passivity theory [18], [19], output regulation [20], incremental stability [21], Lyapunov approach [22], to mention a few recent results. Lur'e systems constitute a popular benchmark for testing these theories [23]-[26]. Here we employ the IOS theory to derive conditions for robust synchronization (in the presence of external inputs).

The outline of this paper is as follows. In Section II, the stability definitions are presented. The class of considered systems is defined in Section III, and in Section IV, the conditions for IOS and the analysis of the selected Lyapunov function are given. The synchronization measure and an approach to study synchronization of a family of generalized Persidskii systems are introduced in Section V-A. Robust control design for synchronization of linear systems subject to highly nonlinear perturbations is presented in Section V-B. The HindmarshRose model is considered as an example in Section VI to examine the efficiency of our proposed results.

\section{Notation}

- $\mathbb{N}, \mathbb{R}$, and $\mathbb{R}_{+}$represent the sets of natural numbers, real numbers, and nonnegative real numbers, respectively. The symbols $|\cdot|$ and $\|\cdot\|$ denote the absolute value in $\mathbb{R}$ and the Euclidean norm on $\mathbb{R}^{n}$ (and the induced matrix norm $\|A\|$ for a matrix $A \in \mathbb{R}^{m \times n}$ ), correspondingly.

- The identity matrix of dimension $n$ is denoted by $I_{n}$ and the $n$-dimensional all-ones vector by $\mathbf{1}_{n}$. The set of diagonal matrices with nonnegative elements on the main diagonal is denoted by $\mathbb{D}_{+}^{n} \subset \mathbb{R}_{+}^{n \times n}$. For a symmetric matrix $A \in \mathbb{R}^{n \times n}, \lambda_{\max }(A)$ denotes its maximal eigenvalue. For a matrix $B \in \mathbb{R}^{m \times n}$, let $\operatorname{ker}(B)$ denote its kernel.

- For $p, n \in \mathbb{N}$ with $p \leq n$, the notation $\overline{p, n}$ is used to represent the set $\{p, \ldots, n\}$.

- For a Lebesgue measurable function $u: \mathbb{R}_{+} \rightarrow \mathbb{R}^{m}$, define the norm $\|u\|_{\left[t_{1}, t_{2}\right)}=\operatorname{ess~sup}_{t \in\left[t_{1}, t_{2}\right)}\|u(t)\|$ for $\left[t_{1}, t_{2}\right) \subset \mathbb{R}_{+}$. Let $\mathscr{L}_{\infty}^{m}$ be the space of functions $u$ with $\|u\|_{\infty}:=$ $\|u\|_{[0, \infty)}<+\infty$ and $\tilde{\mathscr{L}}_{\Theta}^{m} \subset \mathscr{L}_{\infty}^{m}$ be the space of functions taking values in a compact subset $\Theta \subset \mathbb{R}^{m}$.

- A continuous function $\sigma: \mathbb{R}_{+} \rightarrow \mathbb{R}_{+}$belongs to class $\mathscr{K}$ if it is strictly increasing and $\sigma(0)=0$; it belongs to class $\mathscr{K}_{\infty}$ if it also satisfies $\lim _{r \rightarrow \infty} \sigma(r)=\infty$. A continuous function $\beta: \mathbb{R}_{+} \times \mathbb{R}_{+} \rightarrow \mathbb{R}_{+}$belongs to class $\mathscr{K} \mathscr{L}$ if for fixed $s \in \mathbb{R}_{+}, \beta(\cdot, s) \in \mathscr{K}$ and for fixed $r \in \mathbb{R}_{+}, \beta(r, \cdot)$ is a decreasing function with $\lim _{s \rightarrow \infty} \beta(r, s)=0$. 
- For a continuously differentiable function $V: \mathbb{R}^{n} \rightarrow \mathbb{R}$, denote by $\nabla V(v) f(v)$ the Lie derivative of $V$ along the vector field $f$ evaluated at point $v \in \mathbb{R}^{n}$.

\section{PRELIMINARIES}

Consider a class of nonlinear systems:

$$
\begin{aligned}
& \dot{x}(t)=f(x(t), d(t)), \forall t \geq 0, \text { with } f(0,0)=0, x(0)=x_{0}, \\
& y(t)=h(x(t)),
\end{aligned}
$$

where $x(t) \in \mathbb{R}^{n}$ is the state vector, $d(t) \in \mathbb{R}^{m}$ is the external perturbation, $d \in \mathscr{L}_{\infty}^{m}, y(t) \in \mathbb{R}^{p}$ is the output vector. Moreover, $f: \mathbb{R}^{n} \times \mathbb{R}^{m} \rightarrow \mathbb{R}^{n}$ is a locally Lipschitz continuous function and $h: \mathbb{R}^{n} \rightarrow \mathbb{R}^{p}$ is a continuously differentiable function. For an initial state $x_{0} \in \mathbb{R}^{n}$ and $d \in \mathscr{L}_{\infty}^{m}$, the corresponding solution of (1) is denoted by $x\left(t, x_{0}, d\right)$ for the values of $t \geq 0$ the solution exists, so the corresponding output is $y\left(t, x_{0}, d\right)=h\left(x\left(t, x_{0}, d\right)\right)$.

The system (1) is forward complete if for all $x_{0} \in \mathbb{R}^{n}$ and $d \in \mathscr{L}_{\infty}^{m}$, the solution $x\left(t, x_{0}, d\right)$ is uniquely defined for all $t \geq 0$.

In the rest of the paper, to lighten the notation, the timedependency of variables might remain implicitly understood, for instance we write $x$ for $x(t)$. Let us give some definitions that are used in the sequel.

Definition 1. [6] A forward complete system (1) is said to be:

1) practical input-to-output stable (pIOS) if there exist $\beta \in$ $\mathscr{K} \mathscr{L}, \gamma \in \mathscr{K}$ and $c \in \mathbb{R}_{+}$such that

$$
\left\|y\left(t, x_{0}, d\right)\right\| \leq \beta\left(\left\|x_{0}\right\|, t\right)+\gamma\left(\|d\|_{\infty}\right)+c, \forall t \geq 0
$$

for any $x_{0} \in \mathbb{R}^{n}$ and $d \in \mathscr{L}_{\infty}^{m}$. The system is input-tooutput stable $(I O S)$ if $c=0$. When $y=x$, the IOS property is called input-to-state stability (ISS).

2) output-Lagrange input-to-output stable (OLIOS) if it is IOS and there exist $\sigma_{1}, \sigma_{2} \in \mathscr{K}$ such that

$$
\left\|y\left(t, x_{0}, d\right)\right\| \leq \max \left\{\sigma_{1}\left(\left\|h\left(x_{0}\right)\right\|\right), \sigma_{2}\left(\|d\|_{\infty}\right)\right\}, \forall t \geq 0
$$

for any $x_{0} \in \mathbb{R}^{n}$ and $d \in \mathscr{L}_{\infty}^{m}$.

3) state-independent input-to-output stable (SIIOS) if there exist $\beta \in \mathscr{K} \mathscr{L}, \gamma \in \mathscr{K}$ such that

$$
\left\|y\left(t, x_{0}, d\right)\right\| \leq \beta\left(\left\|h\left(x_{0}\right)\right\|, t\right)+\gamma\left(\|d\|_{\infty}\right), \forall t \geq 0
$$

for any $x_{0} \in \mathbb{R}^{n}$ and $d \in \mathscr{L}_{\infty}^{m}$.

4) robustly output stable (ROS) if there exist a smooth function $\alpha \in \mathscr{K}_{\infty}$ and $\beta \in \mathscr{K} \mathscr{L}$ such that the system

$$
\dot{x}=\tilde{f}(x, \varsigma):=f(x, \varsigma \alpha(\|h(x)\|))
$$

is forward complete, and the estimate

$$
\left\|y_{\alpha}\left(t, x_{0}, \varsigma\right)\right\| \leq \beta\left(\left\|x_{0}\right\|, t\right), \forall t \geq 0
$$

is satisfied for all $\varsigma \in \tilde{\mathscr{L}}_{\mathscr{C}}^{m}$, where $\mathscr{C}:=\left\{\mu \in \mathbb{R}^{m}:\|\mu\| \leq\right.$ $1\}$, and $y_{\alpha}\left(t, x_{0}, \varsigma\right)=h\left(x\left(t, x_{0}, \varsigma\right)\right)$ denotes the output function of the system (2).

Definition 2. [6] A forward complete system (1) is said to be uniformly bounded-input-bounded-state stable (UBIBS) if there exists $\sigma \in \mathscr{K}$ such that

$$
\left\|x\left(t, x_{0}, d\right)\right\| \leq \max \left\{\sigma\left(\left\|x_{0}\right\|\right), \sigma\left(\|d\|_{\infty}\right)\right\}, \forall t \geq 0
$$

for all $x_{0} \in \mathbb{R}^{n}$ and $d \in \mathscr{L}_{\infty}^{m}$.

Definition 3. [6] For the system (1), a smooth function $V: \mathbb{R}^{n} \rightarrow \mathbb{R}_{+}$is:

1) an IOS-Lyapunov function if there exist $\alpha_{1}, \alpha_{2} \in \mathscr{K}_{\infty}$, $\chi \in \mathscr{K}$ and $\alpha_{3} \in \mathscr{K} \mathscr{L}$ such that

$$
\begin{gathered}
\alpha_{1}(\|h(x)\|) \leq V(x) \leq \alpha_{2}(\|x\|), \\
V(x) \geq \chi(\|d\|) \Rightarrow \nabla V(x) f(x, d) \leq-\alpha_{3}(V(x),\|x\|)
\end{gathered}
$$

for all $x \in \mathbb{R}^{n}$ and $d \in \mathbb{R}^{m}$.

2) an OLIOS-Lyapunov function if there exist $\alpha_{1}, \alpha_{2} \in \mathscr{K}_{\infty}$, $\chi \in \mathscr{K}$ and $\alpha_{3} \in \mathscr{K} \mathscr{L}$ such that

$$
\begin{gathered}
\alpha_{1}(\|h(x)\|) \leq V(x) \leq \alpha_{2}(\|h(x)\|), \\
V(x) \geq \chi(\|d\|) \Rightarrow \nabla V(x) f(x, d) \leq-\alpha_{3}(V(x),\|x\|)
\end{gathered}
$$

for all $x \in \mathbb{R}^{n}$ and $d \in \mathbb{R}^{m}$.

3) an SIIOS-Lyapunov function if there exist $\alpha_{1}, \alpha_{2} \in \mathscr{K}_{\infty}$ and $\chi, \alpha_{3} \in \mathscr{K}$ such that

$$
\begin{gathered}
\alpha_{1}(\|h(x)\|) \leq V(x) \leq \alpha_{2}(\|h(x)\|), \\
V(x) \geq \chi(\|d\|) \Rightarrow \nabla V(x) f(x, d) \leq-\alpha_{3}(V(x))
\end{gathered}
$$

for all $x \in \mathbb{R}^{n}$ and $d \in \mathbb{R}^{m}$.

4) an $R O S$-Lyapunov function if there exist $\alpha_{1}, \alpha_{2} \in \mathscr{K}_{\infty}$, $\chi \in \mathscr{K}$ and $\alpha_{3} \in \mathscr{K} \mathscr{L}$ such that

$$
\begin{gathered}
\alpha_{1}(\|h(x)\|) \leq V(x) \leq \alpha_{2}(\|x\|), \\
\|h(x)\| \geq \chi(\|d\|) \Rightarrow \nabla V(x) f(x, d) \leq-\alpha_{3}(V(x),\|x\|)
\end{gathered}
$$

for all $x \in \mathbb{R}^{n}$ and $d \in \mathbb{R}^{m}$.

Theorem 1. [6] A UBIBS system (1) is IOS (OLIOS, SIIOS, ROS) if and only if it admits an IOS (OLIOS, SIIOS, ROS)Lyapunov function.

Remark 1. Note that for a sufficient condition of IOS, SIIOS, or ROS, the UBIBS requirement can be discarded provided that the system (1) is forward complete (or it possesses the unboundedness observability property [27]) and an IOS/SIIOSLyapunov function or a ROS-Lyapunov function satisfies (3) ((4) in SIIOS case) and respectively,

$$
V(x) \geq \chi(\|d\|) \Rightarrow \nabla V(x) f(x, d) \leq-\alpha_{3}(V(x))
$$

or

$$
\|h(x)\| \geq \chi(\|d\|) \Rightarrow \nabla V(x) f(x, d) \leq-\alpha_{3}(V(x))
$$

for all $x \in \mathbb{R}^{n}$ and $d \in \mathbb{R}^{m}$, some $\alpha_{1}, \alpha_{2} \in \mathscr{K}_{\infty}$ and $\chi, \alpha_{3} \in \mathscr{K}$.

\section{PRoblem Statement}

Consider the following class of systems:

$$
\begin{aligned}
& \dot{x}(t)=A_{0} x(t)+\sum_{j=1}^{M} A_{j} f^{j}(x(t))+d(t), \forall t \geq 0, \\
& y(t)=C x(t),
\end{aligned}
$$

where $x(t)=\left[x_{1}(t) \ldots x_{n}(t)\right]^{\top} \in \mathbb{R}^{n}$ is the state vector, $y(t) \in$ $\mathbb{R}^{p}$ is the output signal, $C \in \mathbb{R}^{p \times n}$ with $C \neq 0, d \in \mathscr{L}_{\infty}^{n}$ is the external perturbation, $f^{j}: \mathbb{R}^{n} \rightarrow \mathbb{R}^{n}$ with $f^{j}(x)=$ $\left[f_{1}^{j}\left(x_{1}\right) \ldots f_{n}^{j}\left(x_{n}\right)\right]^{\top}, j \in \overline{1, M}(M \in \mathbb{N} \backslash\{0\})$ are continuous 
functions ensuring the existence of solutions of (6) in the forward time at least locally and $A_{k} \in \mathbb{R}^{n \times n}, k \in \overline{0, M}$.

In this paper, it is assumed that if the upper limit of a summation or a sequence is smaller than the lower one, then the corresponding terms (or conditions) are omitted.

Assumption 1. For any $i \in \overline{1, n}, j \in \overline{1, M}$ :

$$
\tau f_{i}^{j}(\tau)>0, \quad \forall \tau \in \mathbb{R} \backslash\{0\} .
$$

This assumption states that all nonlinearities belong to a sector and may take zero values at zero only, and it is the main restriction on the class of systems given in (6).

For further use, we denote by the index $m \in \overline{0, M}$, a positive integer such that for all $i \in \overline{1, n}, a \in \overline{1, m}$ :

$$
\lim _{\tau \rightarrow \pm \infty} f_{i}^{a}(\tau)= \pm \infty
$$

and by $\mu \in \overline{m, M}$, a positive integer such that for all $i \in \overline{1, n}$, $b \in \overline{1, \mu}$ :

$$
\lim _{v \rightarrow \pm \infty} \int_{0}^{v} f_{i}^{b}(\tau) d \tau=+\infty .
$$

The index $m>0$ characterizes the radially unbounded nonlinearities, and $m=0$ corresponds to the case when all nonlinearities are bounded (at least for negative or positive arguments). The index $\mu>0$ selects the nonlinearities having unbounded integrals. Clearly, if $m>0$, then all radially unbounded nonlinearities also have unbounded integrals, thus $\mu \geq m$ due to the introduced sector condition. Indexes $m$ and $\mu$ can be obtained after a proper re-indexing and decomposition of the $f^{j}$, and the featured restriction of (6) is formulated in Assumption 1 (the sector condition).

Remark 2. The Lur'e models under the sector conditions [28], [29] may be presented in the form (6) under Assumption 1. The advantage of (6) over Lur'e dynamics is that all cross-terms between $x_{i}$ and $f_{i}^{j}\left(x_{i}\right)$ appearing in the expressions of $V$ and $\dot{V}$ can be accurately treated, rather than be considered as perturbations (see [13] or the next section). The same analysis in the conventional form of Lur'e model can be less straightforward (especially for $M>1$ and for $f_{i}^{j}\left(x_{i}\right) f_{i}^{k}\left(x_{i}\right)$ with $\left.j \neq k \in \overline{1, M}\right)$.

The principal goals of this work are to propose an approach to check IOS, ROS, and SIIOS properties for the generalized Persidskii system in (6), to apply the obtained conditions for the synchronization analysis in a family of systems as (6), and to design a nonlinear robust synchronization control in this framework.

\section{IOS CONDITIONS}

The main result of this paper is as follows:

Theorem 2. Let Assumption 1 be satisfied. If there exist $0 \leq$ $P_{1}=P_{1}^{\top} \in \mathbb{R}^{p \times p}, 0 \leq P_{2}=P_{2}^{\top} \in \mathbb{R}^{n \times n}, \Lambda^{j}=\operatorname{diag}\left(\Lambda_{1}^{j}, \ldots, \Lambda_{n}^{j}\right) \in$ $\mathbb{D}_{+}^{n}$ for $j \in \overline{1, M}, \Theta \in \mathbb{D}_{+}^{n}, \Psi \in \mathbb{D}_{+}^{p}, \Xi^{k} \in \mathbb{D}_{+}^{n}$ for $k \in \overline{0, M}, \Upsilon_{s, z} \in$ $\mathbb{D}_{+}^{n}$ for $s \in \overline{0, M-1}, z \in \overline{s+1, M}$ and $0<\Phi=\Phi^{\top} \in \mathbb{R}^{n \times n}$ such that

$$
\begin{gathered}
P_{1}>0 \text { or } P_{2}>0 \text { or } \sum_{j=1}^{\mu} \Lambda^{j}>0 ; \\
P_{2} \leq \Theta, Q \leq 0,
\end{gathered}
$$

where

$$
\begin{aligned}
& Q=\left[\begin{array}{cccccc}
Q_{1,1} & Q_{1,2} & Q_{1,3} & \ldots & Q_{1, M+1} & P_{C} \\
Q_{1,2}^{\top} & Q_{2,2} & Q_{2,3} & \ldots & Q_{2, M+1} & \Lambda^{1} \\
Q_{1,3}^{\top} & Q_{2,3}^{\top} & Q_{3,3} & \ldots & Q_{3, M+1} & \Lambda^{2} \\
\vdots & \vdots & \vdots & \ddots & \vdots & \vdots \\
Q_{1, M+1}^{\top} & Q_{2, M+1}^{\top} & Q_{3, M+1}^{\top} & \ldots & Q_{M+1, M+1} & \Lambda^{M} \\
P_{C} & \Lambda^{1} & \Lambda^{2} & \ldots & \Lambda^{M} & -\Phi
\end{array}\right] \\
& P_{C}=C^{\top} P_{1} C+P_{2} ; \quad Q_{1,1}=A_{0}^{\top} P_{C}+P_{C} A_{0}+\Xi^{0}+C^{\top} \Psi C ; \\
& Q_{j+1, j+1}=A_{j}^{\top} \Lambda^{j}+\Lambda^{j} A_{j}+\Xi^{j}, j \in \overline{1, M} ; \quad Q_{1, j+1}=P_{C} A_{j}+A_{0}^{\top} \Lambda^{j}+\Upsilon_{0, j}, j \in \overline{1, M} ; \\
& Q_{S+1, z+1}=A_{S}^{\top} \Lambda^{z}+\Lambda^{s} A_{z}+\Upsilon_{S, z}, s \in \overline{1, M-1}, z \in \overline{s+1, M},
\end{aligned}
$$

then a forward complete system (6) is ROS if

$$
\Psi>0, \Theta+\sum_{j=1}^{M} \Lambda^{j} \leq \xi\left(\sum_{k=0}^{M} \Xi^{k}+2 \sum_{s=0}^{M-1} \sum_{z=s+1}^{M} \Upsilon_{s, z}\right),
$$

or IOS if

$$
P_{1} \leq \xi \Psi, \Theta+\sum_{j=1}^{M} \Lambda^{j} \leq \xi\left(\sum_{k=0}^{m} \Xi^{k}+2 \sum_{s=0}^{m-1} \sum_{z=s+1}^{m} \Upsilon_{s, z}\right)
$$

for some $\xi>0$.

Proof. Consider a candidate Lyapunov function

$$
V(x)=x^{\top} P_{C} x+2 \sum_{j=1}^{M} \sum_{i=1}^{n} \Lambda_{i}^{j} \int_{0}^{x_{i}} f_{i}^{j}(\tau) d \tau .
$$

If $P_{1}>0$, then

$$
y^{\top} P_{1} y \leq V(x) \leq \alpha_{2}(\|x\|)
$$

with $\alpha_{2}(\tau) \leq \lambda_{\max }\left(P_{C}\right) \tau^{2}+2 n M \max _{i \in \overline{1, n}, j \in \overline{1, M}}\left\{\Lambda_{i}^{j} \int_{0}^{\tau} f_{i}^{j}(\gamma) d \gamma\right\}$

a function from class $\mathscr{K}_{\infty}$, so (3) is verified. If instead, $P_{2}>0$ or $\sum_{j=1}^{\mu} \Lambda^{j}>0($ see $(7))$, then $\alpha_{1}(\|x\|) \leq V(x) \leq \alpha_{2}(\|x\|)$ for a function $\alpha_{1} \in \mathscr{K}_{\infty}$ (due to Assumption 1) and the definition of $\mu$. Since $\|y\| \leq\|C\|\|x\|$ with $C \neq 0$, then (3) is again satisfied. Consider the derivative of $V$ calculated for (6):

$$
\begin{aligned}
\dot{V}= & \dot{x}^{\top} P_{C} x+x^{\top} P_{C} \dot{x}+2 \sum_{j=1}^{M} \sum_{i=1}^{n} \Lambda_{i}^{j} f_{i}^{j}\left(x_{i}\right) \dot{x}_{i} \\
= & x^{\top}\left(A_{0}^{\top} P_{C}+P_{C} A_{0}\right) x+\left(\sum_{j=1}^{M} f^{j}(x)^{\top} A_{j}^{\top}\right) P_{C} x \\
& +x^{\top} P_{C} \sum_{j=1}^{M} A_{j} f^{j}(x)+2 x^{\top} P_{C} d \\
& +2 \sum_{j=1}^{M}\left(x^{\top} A_{0}^{\top}+d^{\top}+\left(\sum_{s=1}^{M} f^{s}(x)^{\top} A_{s}^{\top}\right)\right) \Lambda^{j} f^{j}(x) .
\end{aligned}
$$


Therefore, under (7) we obtain

$$
\begin{aligned}
\dot{V}= & {\left[\begin{array}{c}
x \\
f^{1}(x) \\
\vdots \\
f^{M}(x) \\
d
\end{array}\right]^{\top} Q\left[\begin{array}{c}
x \\
f^{1}(x) \\
\vdots \\
f^{M}(x) \\
d
\end{array}\right]-x^{\top}\left(C^{\top} \Psi C+\Xi^{0}\right) x } \\
& -\sum_{j=1}^{M} f^{j}(x)^{\top} \Xi^{j} f^{j}(x)-2 \sum_{j=1}^{M} x^{\top} \Upsilon_{0, j} f^{j}(x) \\
& -2 \sum_{s=1}^{M-1} \sum_{z=s+1}^{M} f^{s}(x)^{\top} \Upsilon_{s, z} f^{z}(x)+d^{\top} \Phi d \\
\leq & -x^{\top}\left(C^{\top} \Psi C+\Xi^{0}\right) x-2 \sum_{s=1}^{M-1} \sum_{z=s+1}^{M} f^{s}(x)^{\top} \Upsilon_{s, z} f^{z}(x) \\
& -\sum_{j=1}^{M} f^{j}(x)^{\top} \Xi^{j} f^{j}(x)-2 \sum_{j=1}^{M} x^{\top} \Upsilon_{0, j} f^{j}(x)+d^{\top} \Phi d .
\end{aligned}
$$

If $\Psi>0$, then under the restriction $\frac{1}{2} y^{\top} \Psi y \geq d^{\top} \Phi d$ we conclude that

$$
\begin{aligned}
\dot{V} \leq & -x^{\top}\left(\frac{1}{2} C^{\top} \Psi C+\Xi^{0}\right) x-\sum_{j=1}^{M} f^{j}(x)^{\top} \Xi^{j} f^{j}(x) \\
& -2 \sum_{j=1}^{M} x^{\top} \Upsilon_{0, j} f^{j}(x)-2 \sum_{s=1}^{M-1} \sum_{z=s+1}^{M} f^{s}(x)^{\top} \Upsilon_{s, z} f^{z}(x) .
\end{aligned}
$$

Now we have to show that there exists $\alpha \in \mathscr{K}$ such that

$$
\begin{gathered}
\alpha(V(x)) \leq x^{\top}\left(\frac{1}{2} C^{\top} \Psi C+\Xi^{0}\right) x+\sum_{j=1}^{M} f^{j}(x)^{\top} \Xi^{j} f^{j}(x) \\
+2 \sum_{j=1}^{M} x^{\top} \Upsilon_{0, j} f^{j}(x)+2 \sum_{s=1}^{M-1} \sum_{z=s+1}^{M} f^{s}(x)^{\top} \Upsilon_{s, z} f^{z}(x),
\end{gathered}
$$

which is true taking into account the form of $V$ and if

$$
P_{1} \leq \xi \Psi, \Theta+\sum_{j=1}^{M} \Lambda^{j} \leq \xi\left(\sum_{k=0}^{M} \Xi^{k}+2 \sum_{s=0}^{M-1} \sum_{z=s+1}^{M} \Upsilon_{s, z}\right)
$$

for some $\xi>0$. The latter properties are imposed in the theorem (the first inequality can be verified since $\Psi>0$ ). Hence,

$$
\frac{1}{2} y^{\top} \Psi y \geq d^{\top} \Phi d \Rightarrow \dot{V} \leq-\alpha(V) .
$$

Recalling Remark 1, by Theorem 1, we conclude that the system is ROS. To ensure the IOS property, if the function $\alpha \in \mathscr{K}_{\infty}$ in (11), then the property (5) can be guaranteed:

$$
V \geq \alpha^{-1}\left(2 d^{\top} \Phi d\right) \Rightarrow \dot{V} \leq-\frac{1}{2} \alpha(V),
$$

which according to Theorem 1 and Remark 1 is necessary to substantiate (the condition (3) has been already verified). The function $\alpha$ can be selected in the required class under the introduced conditions (8) since only the first $m$ nonlinearities and the quadratic term are radially unbounded.

Remark 3. In the case that IOS conditions are verified with $P_{2}>0$ or $\sum_{j=1}^{\mu} \Lambda^{j}>0$, the system is UBIBS, and the requirement on forward completeness stated in Theorem 2 can be dropped.
Remark 4. The Lyapunov function (9) was frequently used in the absolute stability theory [28]-[30].

To formulate the conditions of OLIOS or SIIOS for the system (6), note that according to Definition 3, the difference between the corresponding Lyapunov functions is in the form of the function $\alpha_{3}$ only (it can belong to the class $\mathscr{K} \mathscr{L}$ or $\mathscr{K})$. As we can conclude from the proof of Theorem 2, within the applied framework, only $\alpha_{3} \in \mathscr{K}$ can be obtained. Hence, we have to restrict our analysis to the SIIOS case and the following additional hypothesis is needed:

Assumption 2. For any $j \in \overline{1, m}$ :

$$
x^{\top} C^{\top} C f^{j}(x)>0, \forall x \in \mathbb{R}^{n} \backslash\left\{x \in \mathbb{R}^{n}: C x=0\right\} .
$$

The last assumption assumes that all unbounded nonlinearities possess a kind of symmetry that $C f^{j}$ takes zero on the set where $y=0$ only. Such a restriction is satisfied if, for example, $f_{i}^{j}(s)=f_{1}^{j}(s)$ for all $i \in \overline{2, n}$ and $j \in \overline{1, m}$, and $C=\Gamma$ as in (15), under which Assumption 2 is essentially an incremental passivity condition for all nonlinearities [31], [32].

Theorem 3. Let assumptions 1 and 2 be satisfied and $C^{\top} C \in$ $\mathbb{D}_{+}^{n}$. If there exist $0<P_{1}=P_{1}^{\top} \in \mathbb{R}^{p \times p} ; \Lambda^{j}=\operatorname{diag}\left(\Lambda_{1}^{j}, \ldots, \Lambda_{n}^{j}\right) \in$ $\mathbb{D}_{+}^{n}$ with $\operatorname{ker}\left(\Lambda^{j}\right)=\operatorname{ker}(C)$ for $j \in \overline{1, M} ; \Xi^{k} \in \mathbb{D}_{+}^{p}$ for $k \in \overline{0, M}$, $\Upsilon_{s, z} \in \mathbb{D}_{+}^{p}$ for $s \in \overline{0, M-1}, z \in \overline{s+1, M}$; and $0<\Phi=\Phi^{\top} \in \mathbb{R}^{n \times n}$ such that

$$
Q \leq 0,
$$

where the matrix $Q$ is given in Theorem 2 under substitutions $\Psi \rightarrow 0, \Upsilon_{s, z} \rightarrow C^{\top} \Upsilon_{s, z} C$ for $s \in \overline{0, M-1}$ and $z \in \overline{s+1, M}$, $\Xi^{k} \rightarrow C^{\top} \Xi^{k} C$ for $k \in \overline{0, M}$ with $P_{2}=0$, then a forward complete system (6) is SIIOS if for some $\xi>0$ :

$$
P_{1} \leq \xi \Xi^{0}, \sum_{j=1}^{M} \Lambda^{j} \leq \xi C^{\top}\left(\sum_{k=1}^{m} \Xi^{k}+2 \sum_{s=0}^{m-1} \sum_{z=s+1}^{m} \Upsilon_{s, z}\right) C .
$$

Proof. Consider a candidate Lyapunov function

$$
V(x)=x^{\top} C^{\top} P_{1} C x+2 \sum_{j=1}^{M} \sum_{i=1}^{n} \Lambda_{i}^{j} \int_{0}^{x_{i}} f_{i}^{j}(\tau) d \tau,
$$

then it is straightforward that (4) is verified for any $P_{1}>0$ and due to the imposed conditions on the kernels of $\Lambda^{j}$ and $C$. The derivative of $V$ calculated for (6) under the assumptions of the theorem can be upper estimated as follows for $Q \leq 0$ :

$$
\begin{aligned}
\dot{V} \leq & -x^{\top} C^{\top} \Xi^{0} C x-\sum_{j=1}^{M} f^{j}(x)^{\top} C^{\top} \Xi^{j} C f^{j}(x) \\
& -2 \sum_{j=1}^{M} x^{\top} C^{\top} \Upsilon_{0, j} C f^{j}(x) \\
& -2 \sum_{s=1}^{M-1} \sum_{z=s+1}^{M} f^{s}(x)^{\top} C^{\top} \Upsilon_{s, z} C f^{z}(x)+d^{\top} \Phi d
\end{aligned}
$$

and for the condition (12) there exists $\alpha \in \mathscr{K}_{\infty}$ such that

$$
\dot{V} \leq-\alpha(V)+d^{\top} \Phi d,
$$

which according to Theorem 1 and Remark 1 implies in our case SIIOS. 
The conditions of both theorems, 2 and 3, can be combined and also used for stability analysis (with $d(t)=0$ for all $t \geq 0$ ):

Corollary 1. Let assumptions 1 and 2 be satisfied and there exist $0 \leq P_{1}=P_{1}^{\top} \in \mathbb{R}^{p \times p}, 0 \leq P_{2}=P_{2}^{\top} \in \mathbb{R}^{n \times n} ; \Xi^{k} \in \mathbb{D}_{+}^{p}$ for $k \in \overline{0, M} ; \Lambda^{j} \in \mathbb{D}_{+}^{n}$ for $j \in \overline{1, M} ; \Upsilon_{s, z} \in \mathbb{D}_{+}^{p}$ for $s \in \overline{0, M-1}$ and $z \in \overline{s+1, M}$ such that

$$
P_{2}>0 \text { or } \sum_{q=1}^{\mu} \Lambda^{q}>0 ; Q \leq 0
$$

where

$$
\begin{gathered}
Q=\left[\begin{array}{ccccc}
Q_{1,1} & Q_{1,2} & Q_{1,3} & \ldots & Q_{1, M+1} \\
Q_{1,2}^{\top} & Q_{2,2} & Q_{2,3} & \ldots & Q_{2, M+1} \\
Q_{1,3}^{\top} & Q_{2,3}^{\top} & Q_{3,3} & \ldots & Q_{3, M+1} \\
\vdots & \vdots & \vdots & \ddots & \vdots \\
Q_{1, M+1}^{\top} & Q_{2, M+1}^{\top} & Q_{3, M+1}^{\top} & \cdots & Q_{M+1, M+1}
\end{array}\right] ; \\
Q_{1,1}=A_{0}^{\top} P_{C} C^{+P_{C} A_{0}+C^{\top} \Xi^{0} C ;} P_{C}=C^{\top} P_{1} C+P_{2} ; \\
Q_{j+1, j+1}=A_{j}^{\top} \Lambda^{j}+\Lambda^{j} A_{j}+C^{\top} \Xi^{j} C, j \in \overline{1, M} ; \\
Q_{1, j+1}=P_{C} A_{j}+A_{0}^{\top} \Lambda^{j}+C^{\top} \Upsilon_{0, j} C, j \in \overline{1, M} ; \\
Q_{S+1, z+1}=A_{S}^{\top} \Lambda^{z}+\Lambda^{s} A_{z}+C^{\top} \Upsilon_{S, z} C, s \in \overline{1, M-1}, z \in \overline{s+1, M} .
\end{gathered}
$$

Then the system (6) is UBIBS and $\lim _{t \rightarrow+\infty}\left\|y\left(t, x_{0}, 0\right)\right\|=0$ for all $x_{0} \in \mathbb{R}^{n}$ if

$$
\sum_{k=0}^{M} \Xi^{k}+2 \sum_{s=0}^{M-1} \sum_{z=s+1}^{M} \Upsilon_{s, z}>0
$$

Proof. Consider the Lyapunov function (9) with $P_{C}=$ $C^{\top} P_{1} C+P_{2}$. If $P_{2}>0$ or $\sum_{z=1}^{\mu} \Lambda^{z}>0$, then

$$
\alpha_{1}(\|x\|) \leq V(x) \leq \alpha_{2}(\|x\|)
$$

for some functions $\alpha_{1}, \alpha_{2} \in \mathscr{K}_{\infty}$, due to Assumption 1 and the definition of $\mu$. Hence, such a Lyapunov function is positive definite and radially unbounded. The derivative of $V$ calculated for (6) with $d=0$ can be upper estimated as follows for $Q \leq 0$ :

$$
\begin{aligned}
& \dot{V} \leq-x^{\top} C^{\top} \Xi^{0} C x-\sum_{j=1}^{M} f^{j}(x)^{\top} C^{\top} \Xi^{j} C f^{j}(x) \\
& -2 \sum_{j=1}^{M} x^{\top} C^{\top} \Upsilon_{0, j} C f^{j}(x)-2 \sum_{s=1}^{M-1} \sum_{z=s+1}^{M} f^{s}(x)^{\top} C^{\top} \Upsilon_{s, z} C f^{z}(x) .
\end{aligned}
$$

Since $\sum_{k=0}^{M} \Xi^{k}+2 \sum_{s=0}^{M-1} \sum_{z=s+1}^{M} \Upsilon_{s, z}>0$ and due to assumptions 1 and 2 , there exists a function $\alpha \in \mathscr{K}$ such that

$$
\dot{V} \leq-\alpha(\|y\|) \text {. }
$$

The proven properties of $V$ and the fact that $\dot{V} \leq 0$ implies that all solutions of (6) are bounded: $\left\|x\left(t, x_{0}, 0\right)\right\| \leq \alpha_{1} \circ \alpha_{2}^{-1}\left(\left\|x_{0}\right\|\right)$ for all $x_{0} \in \mathbb{R}^{n}$ and all $t \geq 0$. Applying standard LaSalle arguments [4], we obtain $\lim _{t \rightarrow+\infty}\left\|y\left(t, x_{0}, 0\right)\right\|=0$, for all $x_{0} \in \mathbb{R}^{n}$.

Remark 5. A minor modification of the conditions given in this section is needed if

$$
y(t)=\left[\begin{array}{llll}
x(t)^{\top} C_{0}^{\top} & \left(f^{1}(x(t))\right)^{\top} C_{1}^{\top} \quad \ldots \quad\left(f^{M}(x(t))\right)^{\top} C_{M}^{\top}
\end{array}\right]^{\top} .
$$

The concepts of IOS and SIIOS can be used for many analysis and design problems, e.g., for synchronization or estimation, and the former issue is considered below.

\section{SYNCHRONIZATION}

A. Synchronization of a family of generalized Persidskii systems

In this subsection, we consider an application of the previously proposed theory.

1) Family of generalized Persidskii systems: Consider a family of $N \geq 2$ systems of the following form:

$$
\dot{x}_{z}(t)=A_{z, 0} x_{z}(t)+\sum_{j=1}^{M} A_{z, j} f^{j}\left(x_{z}(t)\right)+B_{z} u_{z}(t)
$$

$$
+d_{z}(t), z \in \overline{1, N}, \forall t \geq 0,
$$

where $x_{z}(t)=\left[x_{z, 1}(t) \ldots x_{z, n}(t)\right]^{\top} \in \mathbb{R}^{n}$ is the state vector of a subsystem, $A_{z, s} \in \mathbb{R}^{n \times n}$ for $s \in \overline{0, M}, B_{z} \in \mathbb{R}^{n \times r}$, $u_{z}(t)=\left[u_{z, 1}(t) \ldots u_{z, r}(t)\right]^{\top} \in \mathbb{R}^{r}$ is the controlled input, and $d_{z}(t) \in \mathbb{R}^{n}$ is the external perturbation, $d_{z} \in \mathscr{L}_{\infty}^{n} ; f^{j}\left(x_{z}(t)\right)=$ $\left[f_{1}^{j}\left(x_{z, 1}(t)\right) \ldots f_{n}^{j}\left(x_{z, n}(t)\right)\right]^{\top}$ for $j \in \overline{1, M}$ are the functions ensuring existence of the solutions of the system (13) in the forward time at least locally. The sector restrictions on $f^{j}$, $j \in \overline{1, M}$ are imposed as in Assumption 1 .

In this study, we consider the synchronization of the common dynamics of (13), i.e., a system in the following form:

$$
\dot{X}(t)=A_{0} X(t)+\sum_{j=1}^{M} A_{j} F^{j}(X(t))+B U(t)+d(t),
$$

where $X(t)=\left[x_{1}(t)^{\top} \ldots x_{N}(t)^{\top}\right]^{\top} \in \mathbb{R}^{N n}$ is the state vector, $A_{s}=\operatorname{diag}\left(A_{1, s} \ldots A_{N, s}\right) \in \mathbb{R}^{N n \times N n}$ for $s \in \overline{0, M}, B=$ $\operatorname{diag}\left(B_{1} \ldots B_{N}\right) \in \mathbb{R}^{N n \times N r}, \quad U(t)=\left[u_{1}(t)^{\top} \ldots u_{N}(t)^{\top}\right]^{\top} \in$ $\mathbb{R}^{N r}$ is the controlled input, $d(t)=\left[d_{1}^{\top}(t) \cdots d_{N}^{\top}(t)\right]^{\top} \in$ $\mathbb{R}^{N n}$ is the common perturbation, $d \in \mathscr{L}_{\infty}^{N n} ; F^{j}(X(t))=$ $\left[f^{j}\left(x_{1}(t)\right)^{\top} \ldots f^{j}\left(x_{N}(t)\right)^{\top}\right]^{\top} \in \mathbb{R}^{N n}$ for $j \in \overline{1, M}$. Clearly, the functions $F^{j}, j \in \overline{1, M}$ also satisfy the sector condition. We denote the consensus set of (13) as

$$
\mathscr{W}:=\left\{X \in \mathbb{R}^{N n} \mid x_{i}=x_{1} \text { for } i \in \overline{2, N}\right\}
$$

and we say that (14) is in the synchronous mode if $X(t) \in$ $\mathscr{W}$, for all $t \geq 0$. To quantify the closeness of the system to the synchronous regime, we use a synchronization measure: a continuously differentiable function $\rho: \mathbb{R}^{N n} \rightarrow \mathbb{R}^{N n}$ such that $\rho(X)=0$ implies that $X \in \mathscr{W}$. The presence of the disturbances $d \neq 0$ does not allow the system to be in the synchronous mode.

Then the robust synchronization problem can be set: to design a feedback $U=U(X)$ that renders the system (14) to be IOS with respect to the output $\rho$ and the input $d$. If $d=0$, then such a control $U$ pushes (14) to the synchronous mode.

2) Conditions of synchronization: In this study, the synchronization measure $\rho(X)$ is defined as

$$
\text { where } \Gamma=\left[\begin{array}{ccccc}
-I_{n} & I_{n} & 0 & \cdots & 0 \\
0 & -I_{n} & I_{n} & \cdots & 0 \\
\vdots & \vdots & \ddots & \vdots & \vdots \\
0 & 0 & \cdots & -I_{n} & I_{n} \\
I_{n} & 0 & \cdots & 0 & -I_{n}
\end{array}\right] \in \mathbb{R}^{N n \times N n} \text {. }
$$

Note that due to properties of $F^{j}$, in the synchronization mode $\Gamma F^{j}(X)=0$, for all $j \in \overline{1, M}$ and $X \in \mathscr{W}$, i.e., an analog of Assumption 2 is satisfied for $F^{j}, j \in \overline{1, M}$. 
The feedback to robustly synchronize the system (14) (to stabilize the system (14) in IOS sense) is selected in the form of diffusive coupling:

$$
U=K_{0} \Gamma X+\sum_{j=1}^{M} K_{j} \Gamma F^{j}(X)
$$

with $K_{k} \in \mathbb{R}^{r N \times n N}$ for $k \in \overline{0, M}$ designed below.

Remark 6. The control (16) can also be selected in the form of direct coupling [33]:

$$
U=K_{0} X+\sum_{j=1}^{M} K_{j} F^{j}(X),
$$

i.e., the coupling is diffusive if it is proportional to the synchronization measure $\rho$ as in (16), and it is direct if it is given in the form of a generic state feedback. Both types of coupling can be analyzed in the proposed framework, but for brevity the synchronization conditions are formulated below for the diffusive case only.

Substituting the control (16) into the equations of the system (14) we obtain the following closed-loop dynamics:

$$
\dot{X}(t)=\tilde{A}_{0} X(t)+\sum_{j=1}^{M} \tilde{A}_{j} F^{j}(X(t))+d(t), Y(t)=\Gamma X(t),
$$

where $\tilde{A}_{k}=A_{k}+B K_{k} \Gamma$ for $k \in \overline{0, M}$.

Clearly, the system (17) is in the form (6) and assumptions 1 and 2 are satisfied, then theorems 2 and 3 or Corollary 1 can be directly applied.

Corollary 2. If the IOS conditions of Theorems 2 are satisfied under the substitution of $p \rightarrow n N, n \rightarrow n N, C \rightarrow \Gamma, A_{k} \rightarrow \tilde{A}_{k}$, $k \in \overline{0, M}$, then a forward complete system (17) is robustly synchronized.

Proof. As we remarked above, assumptions 1 and 2 are verified by the system (17), and it is forward complete due to hypotheses of the corollary. Then, the IOS property guarantees boundedness of the synchronization error $\rho$ in the presence of essentially bounded perturbations $d \neq 0$, and asymptotic convergence of the synchronization error to zero for $d=0$ (that corresponds to the achievement of the synchronous mode).

Corollary 3. If the conditions of Corollary 1 are satisfied under the substitution of $p \rightarrow n N, n \rightarrow n N, C \rightarrow \Gamma, A_{k} \rightarrow \tilde{A}_{k}$, $k \in \overline{0, M}$, then the system (17) with $d(t)=0, \forall t \in \mathbb{R}_{+}$reaches the synchronous mode.

Proof. It is a direct consequence of Corollary 1 since assumptions 1 and 2 hold.

\section{B. Robust synchronization of linear systems}

Let us consider how the control gains $K_{k} \in \mathbb{R}^{r N \times n N}$ for $k \in \overline{0, M}$ can be designed to ensure synchronization.

For brevity, in this subsection, we consider the robust synchronization of two linear systems

$$
\dot{x}=\left[\begin{array}{c}
\dot{x}^{1} \\
\dot{x}^{2}
\end{array}\right]=A x+B u+d
$$

where $x^{1}, x^{2} \in \mathbb{R}^{n}$ are the states, $A \in \mathbb{R}^{2 n \times 2 n}, B \in \mathbb{R}^{2 n \times m}, u \in \mathbb{R}^{m}$ is the controlled input, $d \in \mathbb{R}^{2 n}$ is the external perturbation, and we assume two scenarios: either $d \in \mathscr{L}_{\infty}^{2 n}$ or $d$ is a nonlinear function of the state $x$ admitting an upper bound

$$
\|d\|^{2} \leq \sum_{i=1}^{2 n} R_{i}^{0}\left|x_{i}\right|^{2}+R_{i}^{1}\left|x_{i}\right|^{1+\zeta}+R_{i}^{2}\left|x_{i}\right|^{1+\pi},
$$

where $\zeta \in(0,1), \pi>1$ are growth parameters and $R^{s}=$ $\operatorname{diag}\left(R_{1}^{s} \ldots R_{2 n}^{s}\right) \in \mathbb{D}_{+}^{2 n}$ are given matrices for $s \in \overline{0,2}$. In the latter case (18) is a nonlinear system, and if $R^{1} \neq 0$ or $R^{2} \neq 0$, then a linear feedback cannot ensure robust synchronization of this system (in the sense of IOS), while Corollary 2 provides a tool for synchronization of the system (18) with such a disturbance.

For (18) we propose to use a feedback control in the form

$$
u=K_{0} \Gamma x+K_{1} \Gamma f^{1}(x)+K_{2} \Gamma f^{2}(x),
$$

where $K_{0}, K_{1}, K_{2} \in \mathbb{R}^{m \times 2 n}$ are the tuning gains,

$$
\Gamma=\left[\begin{array}{cc}
-I_{n} & I_{n} \\
I_{n} & -I_{n}
\end{array}\right]
$$

is the matrix defining synchronization measure for $N=2$ (in such case we may take $\Gamma=\left[I_{n}-I_{n}\right]$ without losing generality), and $f^{1}, f^{2}$ are the functions following the imposed conditions of the system (6) and Assumption 1:

$$
\begin{aligned}
f_{i}^{1}\left(x_{i}\right) & =\left|x_{i}\right|^{\zeta} \operatorname{sign}\left(x_{i}\right), f_{i}^{2}\left(x_{i}\right)=\left|x_{i}\right|^{\pi} \operatorname{sign}\left(x_{i}\right), \\
f^{j}(x) & =\left[f_{1}^{j}\left(x_{1}\right), \ldots, f_{2 n}^{j}\left(x_{2 n}\right)\right], \forall i \in \overline{1,2 n}, j \in \overline{1,2} .
\end{aligned}
$$

Then the resulting closed-loop system is

$$
\dot{x}=A_{0} x+A_{1} f^{1}(x)+A_{2} f^{2}(x)+d,
$$

where $A_{0}=A+B K_{0} \Gamma, A_{1}=B K_{1} \Gamma$ and $A_{2}=B K_{2} \Gamma$. Using the same arguments as in subsection V-A2, we define the output function, or the synchronization measure, of (20) as

$$
y(t)=\Gamma x(t) .
$$

Applying the Lyapunov function from Theorem 2:

$$
V(x)=x^{\top} P_{\Gamma} x+2 \sum_{z=1}^{2} \sum_{j=1}^{2} \sum_{i=1}^{n} \Lambda_{i}^{j} \int_{0}^{x_{i}^{z}} f_{i}^{j}(\tau) d \tau,
$$

where $P_{\Gamma}=\Gamma^{\top} P_{1} \Gamma+P_{2}$, for the system (20) its derivative is calculated as

$$
\begin{aligned}
\dot{V}(x) & =\left[\begin{array}{c}
x \\
f^{1}(x) \\
f^{2}(x) \\
d
\end{array}\right]^{\top} Q\left[\begin{array}{c}
x \\
f^{1}(x) \\
f^{2}(x) \\
d
\end{array}\right]-x^{\top}\left(\Xi^{0}+\Gamma^{\top} \Psi \Gamma\right) x+\phi d^{\top} d \\
& -2 \sum_{j=1}^{2} x^{\top} \Upsilon_{0, j} f^{j}(x)+2 \sum_{j=1}^{2} x^{\top}\left(P_{\Gamma} A_{j}+A_{0}^{\top} \Lambda^{j}+\Upsilon_{0, j}\right) f^{j}(x),
\end{aligned}
$$

where $\Xi^{0}, \Upsilon_{0, j}, \Lambda^{j}$ are given in the formulation of Theorem 2, $\Phi=\phi I_{2 n}$ and

$$
Q=\left[\begin{array}{cccc}
A_{0}^{\top} P_{\Gamma}+P_{\Gamma} A_{0}+\Xi^{0}+\Gamma^{\top} \Psi \Gamma & 0 & 0 & P_{\Gamma} \\
0 & A_{1}^{\top} \Lambda^{1}+\Lambda^{1} A_{1} & A_{1}^{\top} \Lambda^{2}+\Lambda^{1} A_{2} & \Lambda^{1} \\
0 & A_{2}^{\top} \Lambda^{1}+\Lambda^{2} A_{1} & A_{2}^{\top} \Lambda^{2}+\Lambda^{2} A_{2} & \Lambda^{2} \\
P_{\Gamma} & \Lambda^{1} & \Lambda^{2} & -\phi I_{2 n} n
\end{array}\right]
$$


for some $\phi>0$. For the last term in $\dot{V}$, applying Young's inequality for all cross-terms out the main diagonal:

$$
\begin{aligned}
& x_{i}\left|x_{k}\right|^{\zeta} \operatorname{sign}\left(x_{k}\right) \leq \frac{\left|x_{i}\right|^{1+\zeta}}{1+\zeta}+\frac{\zeta\left|x_{k}\right|^{1+\zeta}}{1+\zeta}, \\
& x_{i}\left|x_{k}\right|^{\pi} \operatorname{sign}\left(x_{k}\right) \leq \frac{\left|x_{i}\right|^{1+\pi}}{1+\pi}+\frac{\pi\left|x_{k}\right|^{1+\pi}}{1+\pi}
\end{aligned}
$$

for any $i \neq k \in \overline{1,2 n}$, we obtain that if

$$
\begin{gathered}
\mathscr{Q} \leq 0, \\
\mathbf{1}_{2 n}^{\top}\left[(1+\zeta) \delta\left(P_{\Gamma} A_{1}+A_{0}^{\top} \Lambda^{1}\right)+\zeta \omega\left(P_{\Gamma} A_{1}+A_{0}^{\top} \Lambda^{1}\right)\right. \\
\left.+\omega^{\top}\left(P_{\Gamma} A_{1}+A_{0}^{\top} \Lambda^{1}\right)+\Upsilon_{0,1}\right] \leq 0, \\
\mathbf{1}_{2 n}^{\top}\left[(1+\pi) \delta\left(P_{\Gamma} A_{2}+A_{0}^{\top} \Lambda^{2}\right)+\pi \omega\left(P_{\Gamma} A_{2}+A_{0}^{\top} \Lambda^{2}\right)\right. \\
\left.+\omega^{\top}\left(P_{\Gamma} A_{2}+A_{0}^{\top} \Lambda^{2}\right)+\Upsilon_{0,2}\right] \leq 0,
\end{gathered}
$$

where $\delta(\mathscr{A})$ denotes the diagonal matrix having the diagonal elements of $\mathscr{A}$, and $\omega(\mathscr{A})$ has zero diagonal elements and absolute values of other elements of $\mathscr{A}$, then

$$
x^{\top}\left(P_{\Gamma} A_{j}+A_{0}^{\top} \Lambda^{j}+\Upsilon_{0, j}\right) f^{j}(x) \leq 0, \forall j \in \overline{1,2}
$$

hence, $\dot{V} \leq-x^{\top}\left(\Xi^{0}+\Gamma^{\top} \Psi \Gamma\right) x-2 \sum_{j=1}^{2} x^{\top} \Upsilon_{0, j} f^{j}(x)+\phi d^{\top} d$.

This allows us to present the main result of this section:

Theorem 4. Given $K_{0}, K_{1}, K_{2} \in \mathbb{R}^{m \times 2 n}, \zeta \in(0,1)$ and $\pi>1$, if there exist $0 \leq P_{1}=P_{1}^{\top} \in \mathbb{R}^{2 n \times 2 n}, 0 \leq P_{2}=P_{2}^{\top} \in \mathbb{R}^{2 n \times 2 n}$, $\Lambda^{j}=\operatorname{diag}\left(\Lambda_{1}^{j}, \ldots, \Lambda_{2 n}^{j}\right) \in \mathbb{D}_{+}^{2 n}$ for $j \in \overline{1,2}, \Theta \in \mathbb{D}_{+}^{2 n}, \Psi \in \mathbb{D}_{+}^{2 n}$, $\Xi^{k} \in \mathbb{D}_{+}^{2 n}$ for $k \in \overline{0,2}, \Upsilon_{s, z} \in \mathbb{D}_{+}^{2 n}$ for $s \in \overline{0,1}, z \in \overline{s+1,2}$ and $\phi>0$ such that

$$
P_{1}>0 \text { or } P_{2}>0 \text { or } \sum_{j=1}^{2} \Lambda^{j}>0 ; P_{2} \leq \Theta,
$$

(22), (23) and (24) are satisfied, then a forward complete system (20) is IOS (robustly synchronized) if

$$
P_{1} \leq \xi \Psi, \Theta+\sum_{j=1}^{2} \Lambda^{j} \leq \xi\left(\Xi^{0}+2 \sum_{j=1}^{2} \Upsilon_{0, j}\right)
$$

for some $\xi>0$. If, additionally,

$$
\Xi^{0}+\Gamma^{\top} \Psi \Gamma>\phi R^{0}, 2 \Upsilon_{0, j}>\phi R^{j}, j=1,2,
$$

then for (19) the system is asymptotically reaching the synchronous mode.

Proof. Assume that there exists a function $\alpha \in \mathscr{K}_{\infty}$ such that

$$
2 \alpha(V) \leq x^{\top}\left(\Xi^{0}+\Gamma^{\top} \Psi \Gamma\right) x+2 \sum_{j=1}^{2} x^{\top} \Upsilon_{0, j} f^{j}(x),
$$

then under the restriction $V(x) \geq \alpha^{-1}\left(\phi d^{\top} d\right)$, we get $\dot{V} \leq$ $-\alpha(V)$.

The selection of $\alpha \in \mathscr{K}_{\infty}$ follows the conditions:

$$
P_{1} \leq \xi \Psi, \Theta+\sum_{j=1}^{2} \Lambda^{j} \leq \xi\left(\Xi^{0}+2 \sum_{j=1}^{2} \Upsilon_{0, j}\right)
$$

for some $\xi>0$. The remaining steps repeat the proof of Theorem 2. If the perturbation $d$ satisfies (19), i.e., $d^{\top} d \leq$ $x^{\top} R^{0} x+x^{\top} R^{1} f^{1}(x)+x^{\top} R^{2} f^{2}(x)$, then for (25) under the same conditions we get that $\dot{V} \leq-\epsilon \alpha(V)$ for some $\epsilon \in(0,1)$, implying global stability and convergence of the output $\Gamma x$ to zero.

\section{APPLICATION}

The Hindmarsh-Rose (HR) model [34] is widely used to investigate chaotic behavior in isolated biological cells and neuronal dynamics (being a compact version of the general case [35]):

$$
\begin{aligned}
\dot{x}_{1} & =a x_{1}^{2}-x_{1}^{3}-x_{2}+x_{3}+d, \\
\dot{x}_{2} & =(a+\alpha) x_{1}^{2}-x_{2}, \\
\dot{x}_{3} & =\mu\left(b x_{1}-x_{3}\right)+u,
\end{aligned}
$$

where $x=\left[\begin{array}{lll}x_{1} & x_{2} & x_{3}\end{array}\right]^{\top} \in \mathbb{R}^{3}$ is the state, $d \in \mathbb{R}$ is the disturbance (equivalently applied current in experiments), $u \in \mathbb{R}$ is the control and $a, \alpha, \mu, b \in \mathbb{R}$. Let $\theta>\frac{1}{4}$ be an auxiliary parameter. Then the system (26) can be rewritten as

$$
\dot{x}=\alpha_{0} x+\alpha_{1} f^{1}(x)+\alpha_{2} f^{2}(x)+\tilde{b} u+\tilde{d},
$$

where $\tilde{b}=\left[\begin{array}{lll}0 & 0 & 1\end{array}\right]^{\top}, \tilde{d}=\left[\begin{array}{lll}d & 0 & 0\end{array}\right]^{\top}$,

$$
\begin{gathered}
\alpha_{0}=\left[\begin{array}{ccc}
-a \theta & -1 & 1 \\
-(a+\alpha) \theta & -1 & 0 \\
\mu b & 0 & -\mu
\end{array}\right], \alpha_{1}=\left[\begin{array}{ccc}
-1-a & 0 & 0 \\
-a-\alpha & 0 & 0 \\
0 & 0 & 0
\end{array}\right], \\
\alpha_{2}=\left[\begin{array}{ccc}
a & 0 & 0 \\
a+\alpha & 0 & 0 \\
0 & 0 & 0
\end{array}\right], f^{1}(x)=\left[\begin{array}{l}
x_{1}^{3} \\
x_{2}^{3} \\
x_{3}^{3}
\end{array}\right], f^{2}(x)=\left[\begin{array}{l}
x_{1}\left(x_{1}^{2}+x_{1}+\theta\right) \\
x_{2}\left(x_{2}^{2}+x_{2}+\theta\right) \\
x_{3}\left(x_{3}^{2}+x_{3}+\theta\right)
\end{array}\right],
\end{gathered}
$$

the new nonlinearities $f^{1}$ and $f^{2}$ satisfy the sector condition given in Assumption 1. Let us set the number of systems in the family $N=2, a=2.8, d=3.1, \alpha=1.6, \mu=10^{-3}, b=9$ and $\theta=0.3$. Therefore, the common dynamics of models (27) is

$$
\dot{X}=A_{0} X+A_{1} F^{1}(X)+A_{2} F^{2}(X)+B U+D,
$$

where

$$
\begin{gathered}
X=\left[\begin{array}{l}
x^{1} \\
x^{2}
\end{array}\right] \in \mathbb{R}^{6}, U=\left[\begin{array}{l}
u^{1} \\
u^{2}
\end{array}\right] \in \mathbb{R}^{2}, F^{j}(X)=\left[\begin{array}{l}
f^{j}\left(x^{1}\right) \\
f^{j}\left(x^{2}\right)
\end{array}\right], \forall j \in \overline{1,2}, \\
B=\left[\begin{array}{ll}
\tilde{b} & 0 \\
0 & \tilde{b}
\end{array}\right], A_{s}=\left[\begin{array}{cc}
\alpha_{s} & 0 \\
0 & \alpha_{s}
\end{array}\right], \forall s \in \overline{0,2}, D=\left[\begin{array}{l}
\tilde{d}^{1} \\
\tilde{d}^{2}
\end{array}\right]
\end{gathered}
$$

and $x^{1}, x^{2} \in \mathbb{R}^{3}$ are the solutions of each of the couples HR models (27). Evidently, the system (28) is in the form (14). Consider a feedback control in the form (16), then $U$ is a vector of scalar controls affecting the HR model to synchronize the system (28), we obtain the closed-loop system in the form (17) $\dot{X}=\left(A_{0}+B K_{0} \Gamma\right) X+\left(A_{1}+B K_{1} \Gamma\right) F^{1}(X)+\left(A_{2}+B K_{2} \Gamma\right) F^{2}(X)+D$. The synchronization measure is selected as (15) with $\Gamma=$ $\left[\begin{array}{ll}I_{3} & -I_{3}\end{array}\right]$. Let

$$
\begin{gathered}
K_{0}=\left[\begin{array}{lll}
0.4283 & 0.4820 & 0.1206 \\
0.5895 & 0.2262 & 0.3846
\end{array}\right], \\
K_{1}=\left[\begin{array}{lll}
0 & 0 & 0.5830 \\
0 & 0 & 0.2518
\end{array}\right], K_{2}=\left[\begin{array}{lll}
0 & 0 & 0.2904 \\
0 & 0 & 0.6171
\end{array}\right],
\end{gathered}
$$

then following Corollary 2, there exist matrices solving the proposed LMIs in Theorem 2. The norm of the difference $e:=$ $x^{1}-x^{2}$ and the state trajectories $x^{1}, x^{2}$ of the closed-loop system with distinct initial states $x^{1}(0)=\left[\begin{array}{lll}0.12 & -0.21 & 0.80\end{array}\right]^{\top}$, 
$x^{2}(0)=\left[\begin{array}{lll}0.41 & 0.91 & 0.88\end{array}\right]^{\top}$ are shown in Fig. 1 and Fig. 2, respectively. The simulation results imply that the system (28) is synchronized by the feedback controller, while each separate subsystem remains oscillating.

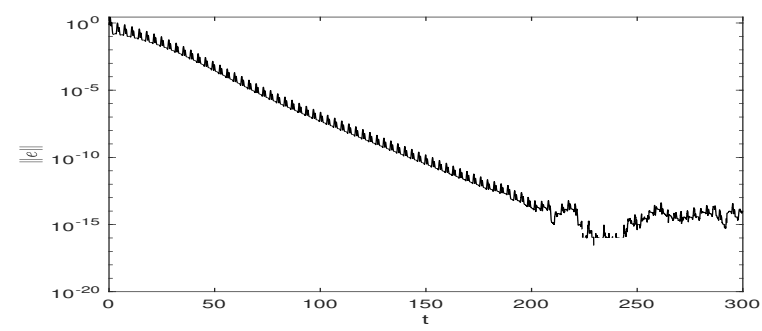

Fig. 1. The norm of the synchronization error $e$ versus the time $t$

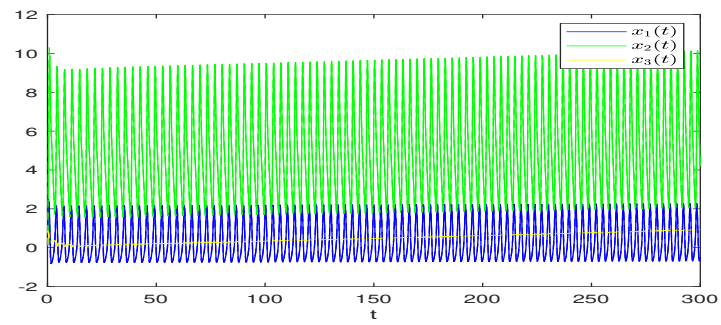

Fig. 2. The state trajectories $x^{1}$ and $x^{2}$ versus the time $t$

\section{CONCLUSION}

In this paper, we proposed IOS, SIIOS, and ROS conditions for generalized Persidskii systems. The conditions were obtained in the form of LMIs. As an application of the developed approach, the problem of the robust synchronization of a family of generalized Persidskii systems was solved. Furthermore, a robust synchronization method was proposed for linear systems with essentially bounded perturbations also admitting a hard upper bound in terms of the state components. The efficiency of the proposed results was illustrated through the synchronization of Hindmarsh-Rose models.

\section{REFERENCES}

[1] W. Mei, D. Efimov, and R. Ushirobira, "Feedback synchronization in Persidskii systems," IFAC-PapersOnLine, vol. 53, pp. 2880-2884, 2020.

[2] M. Vidyasagar, Input-output analysis of large-scale interconnected systems, ser. Lecture Notes in Control and Information Sciences. Berlin: Springer-Verlag, 1981, vol. 29.

[3] A. van der Schaft, $L_{2}$-gain and passivity techniques in nonlinear control, ser. Lecture Notes in Control and Information Sciences. London: Springer-Verlag London Ltd., 1996, vol. 218.

[4] H. Khalil, Nonlinear Systems. Upper Saddle River, NJ: Prentice Hall, 2002.

[5] E. D. Sontag and Y. Wang, "Notions of input to output stability," Systems Control Lett., vol. 38, no. 4-5, pp. 235-248, 1999.

[6] E. Sontag and Y. Wang, "Lyapunov characterizations of input to output stability," SIAM Journal on Control and Optimization, vol. 39, no. 1, pp. 226-249, 2000.

[7] S. Dashkovskiy, D. Efimov, and E. Sontag, "Input to state stability and allied system properties," Automation and Remote Control, vol. 72, no. 8, pp. 1579-1614, 2011.

[8] C. Guiver and H. Logemann, "A circle criterion for strong integral inputto-state stability," Automatica, vol. 111, p. 108641, 2020.

[9] S. Persidskii, "Concerning problem of absolute stability," Automation and Remote Control, vol. 30, no. 12, pp. 1889-1895, 1970.
[10] L. Ferreira, E. Kaszkurewicz, and A. Bhaya, "Solving systems of linear equations via gradient systems with discontinuous righthand sides: Application to LS-SVM," IEEE Transactions on Neural Networks, vol. 16, pp. 501-505, 2005.

[11] L. Hsu and L. Colvara, "The inclusion of automatic voltage regulators in power system transient stability analysis using Lyapunov functions," IFAC Proceedings Volumes, vol. 20, pp. 1-6, 1987.

[12] E. Barbashin, "On construction of Lyapunov functions for nonlinear systems," in Proc. 1st IFAC World Congress, Moscow, 1961, pp. 742751.

[13] D. Efimov and A. Y. Aleksandrov, "Robust stability analysis and implementation of Persidskii systems," in 2019 IEEE 58th Conference on Decision and Control (CDC). IEEE, 2019, pp. 6164-6168.

[14] L. M. Pecora and T. L. Carroll, "Synchronization in chaotic systems," Phys. Rev. Lett., vol. 64, no. 8, pp. 821-824, 1990.

[15] S. H. Strogatz, "From Kuramoto to Crawford: exploring the onset of synchronization in populations of coupled oscillators," Physica D: Nonlinear Phenomena, vol. 143, no. 1, pp. 1-20, 2000.

[16] A. Pikovsky, M. Rosenblum, and J. Kurths, Synchronization: A Universal Concept in Nonlinear Sciences. Cambridge: Cambridge University Press, 2001.

[17] R. Olfati-Saber, J. Fax, and R. Murray, "Consensus and cooperation in networked multi-agent systems," Proceedings of the IEEE, vol. 95, pp. 215-233, 2007

[18] C. D. Persis and B. Jayawardhana, "Coordination of passive systems under quantized measurements," SIAM Journal on Control and Optimization, vol. 50, pp. 3155-3177, 2012.

[19] A. Hamadeh, G. Stan, R. Sepulchre, and J. Goncalves, "Global state synchronization in networks of cyclic feedback systems," IEEE Transactions on Automatic Control, vol. 57, pp. 478-483, 2012.

[20] C. Byrnes, "Uniform output regulation of nonlinear systems: A convergent dynamics approach," IEEE Transactions on Automatic Control, vol. 52, pp. 2013-2014, 2007.

[21] D. Angeli, "Further results on incremental input-to-state stability," IEEE Transactions on Automatic Control, vol. 54, pp. 1386-1391, 2009.

[22] B. T. Polyak and Y. I. Kvinto, "Stability and synchronization of oscillators: New Lyapunov functions," Automation and Remote Control, vol. 78, pp. 1234-1242, 2017.

[23] J. Suykens, P. Curran, and L. Chua, "Robust synthesis for master-slave synchronization of Lur'e systems," IEEE Transactions on Circuits and Systems I: Fundamental Theory and Applications, vol. 46, pp. 841-850, 1999.

[24] F. Zhang, H. L. Trentelman, and J. M. Scherpen, "Fully distributed robust synchronization of networked Lur'e systems with incremental nonlinearities," Automatica, vol. 50, pp. 2515-2526, 2014.

[25] H. Kim and C. De Persis, "Output synchronization of Lur'e-type nonlinear systems in the presence of input disturbances," in 2015 54th IEEE Conference on Decision and Control (CDC). IEEE, 2015, pp. $4145-4150$

[26] A. V. Proskurnikov and M. Cao, "Synchronization of Goodwin's oscillators under boundedness and nonnegativeness constraints for solutions," IEEE Transactions on Automatic Control, vol. 62, pp. 372-378, 2017.

[27] D. Angeli and E. Sontag, "Forward completeness, unboundedness observability, and their Lyapunov characterizations," Systems \& Control Letters, vol. 38, pp. 209-217, 1999.

[28] V. Yakubovich, "Popov's method and its subsequent development," European Journal of Control, vol. 8, no. 3, pp. 200-208, 2002.

[29] M. R. Liberzon, "Essays on the absolute stability theory," Automation and Remote Control, vol. 67, no. 10, pp. 1610-1644, 2006.

[30] V. Yakubovich, "Frequency conditions for the absolute stability of control systems with several nonlinear or linear nonstationary blocks," Avtomat. $i$ Telemekh., no. 6, pp. 5-30, 1967.

[31] A. Pavlov and L. Marconi, "Incremental passivity and output regulation," Systems \& Control Letters, vol. 57, pp. 400-409, 2008.

[32] F. Zhang, H. L. Trentelman, and J. M. A. Scherpen, "Robust cooperative output regulation of heterogeneous Lur'e networks," International Journal of Robust and Nonlinear Control, vol. 27, pp. 3061-3078, 2017.

[33] S. A. Plotnikov and A. L. Fradkov, "On synchronization in heterogeneous FitzHugh-Nagumo networks," Chaos, Solitons \& Fractals, vol. 121 , pp. 85-91, 2019

[34] J. L. Hindmarsh and R. M. Rose, "A model of neuronal bursting using three coupled first order differential equations," Proceedings of the Royal Society of London. Series B. Biological Sciences, vol. 221, pp. 87-102, 1984.

[35] A. L. Hodgkin and A. F. Huxley, "A quantitative description of membrane current and its application to conduction and excitation in nerve," The Journal of Physiology, vol. 117, pp. 500-544, 1952. 